\title{
SIMPLE NONLINEAR DUAL CONTROL PROBLEMS
}

\author{
J. M. MURRAY ${ }^{1}$
}

(Received 21 June 1984; revised 23 November 1984)

\begin{abstract}
In this paper we consider a simple, nonlinear optimal control problem with sufficient convexity to enable us to formulate its dual problem. Both primal and dual problems will include constraints on both the states and controls. The constraints in one problem may cause the "optimal" dual states to be discontinuous. However, we will look at conditions under which the presence of constraints does not force discontinuities and the optımal states and costates are absolutely continuous.
\end{abstract}

\section{Introduction}

In this paper, we investigate simple, constrained control problems, where the constraints contain both state and control terms. The primal problem we consider has sufficient convexity to ensure that a dual problem of similar form to the primal problem can be formulated. The difficulties in computing solutions to the primal problem stem from the constraints in the primal problem and also the constraints in the dual problem. The constraints in the primal problem can cause the optimal costates to jump so that they do not satisfy a differential equation and can contain $\delta$-functions or worse. The constraints in the dual problem can lead to a similar situation occurring for the optimal states. To eliminate these possibilities we place conditions on both the primal and dual problems to ensure that the usual growth conditions found in existence theory for optimal solutions in control theory, apply. These eventually lead to easily verified assumptions which guarantee optimal solutions exist for the primal and/or dual problems. The

\footnotetext{
${ }^{1}$ Department of Applied Mathematics, University of New South Wales, P. O. Box 1, Kensington, N.S.W. 2033.

(C) Copyright Australian Mathematical Society 1985, Serial-fee code 0334-2700/85
} 
main results are to be found in Theorem 3, Corollaries 3 and 4 and Proposition 4. We also find that strong duality holds.

Other results in a similar vein are available elsewhere. Murray [6] looks at dual linear problems with some mention of nonlinear, constrained control problems and Grinold [3] and Hanson and Mond [4] consider a similar primal problem to ours, but use the Wolfe dual. More general problems than [3] have also been investigated, for example [1], [2]. This paper is in a different vein to the ahove works in that it uses the results of existence theory from optimal control theory, and the dual problem is not the Wolfe dual.

\section{The primal and dual problems}

The primal problem that we will consider is the following

$$
\underset{x \in \mathscr{A}^{n}, u \in \mathscr{L}^{m}}{\operatorname{minimize}} \Psi_{P}(x, u)=l\left(x\left(t_{0}\right), x\left(t_{1}\right)\right)+\int_{T}[a(t) x(t)+f(t, u(t))] d t
$$

subject to

$$
\begin{gathered}
\dot{x}(t)=A(t) x(t)+B(t) u(t)+c(t), \\
C(t) x(t)+E(t) u(t)+d(t) \geqslant 0, \\
u(t) \geqslant 0,
\end{gathered}
$$

where $f(t, \cdot)$ is convex for each $t \in T=\left[t_{0}, t_{1}\right]$ and is a normal integrand (this implies $f(t, \cdot)$ is lower semicontinuous; see [11] for its other implications), $l$ is convex and lower semicontinuous, and the matrices $A, B, C$ and $E$, and the vectors $a, c$ and $d$ have components that are essentially bounded, Lebesgue measurable functions. We allow both $l$ and $f$ to take the value $+\infty$ which means there can be implicit constraints in the problem. For example if

we have a fixed endpoint problem.

$$
l\left(x\left(t_{0}\right), x\left(t_{1}\right)\right)=\left\{\begin{array}{l}
0 \text { if } x\left(t_{0}\right)=x_{0} \\
+\infty \text { otherwise }
\end{array}\right.
$$

If $x \in \mathscr{A}^{n}$ then $x$ is an $n$-vector of functions that are absolutely continuous and if $u \in \mathscr{L}^{m}$ then $u$ is an $m$-vector of functions that are Lebesgue measurable.

The dual problem we will associate with $(\mathrm{P})$ has the form

$$
\begin{aligned}
& \underset{p \in \mathscr{A}^{n}, w \in \mathscr{L}^{k}, u^{*} \in \mathscr{L}^{m}}{\operatorname{minimize}} \Psi_{D}\left(p, w, u^{*}\right) \\
& =m\left(p\left(t_{0}\right), p\left(t_{1}\right)\right)+\int_{T}\left[p(t) c(t)+w(t) d(t)+f^{*}\left(t, u^{*}(t)\right)\right] d t
\end{aligned}
$$

subject to

$$
\begin{gathered}
\dot{p}(t)=-p(t) A(t)-w(t) C(t)+a(t), \\
p(t) B(t)+w(t) E(t)-u^{*}(t) \leqslant 0, \\
w(t) \geqslant 0,
\end{gathered}
$$


where $f^{*}(t, \cdot)$ is the conjugate function of $f(t, \cdot)$ that is,

$$
f^{*}\left(t, u^{*}\right)=\sup _{u}\left\{u^{*} u-f(t, u)\right\}
$$

and

$$
m\left(p_{0}, p_{1}\right)=\sup _{x_{0}, x_{1}}\left\{p_{0} x_{0}-p_{1} x_{1}-l\left(x_{0}, x_{1}\right)\right\} .
$$

Our dual is of the type expounded by Rockafellar [8]. This is not the only dual problem one can connect with (P). The Wolfe dual is often investigated, for instance in [3] and [4], where their primal problems have some similarities to ours.

If we take $f(t, u)=b(t) u$ we see that (P) has the same form as problem (P) of [6]. There we had to consider extended primal and dual problems where the controls $u$ and $w$ become measures and $x$ and $p$ were allowed to be functions of bounded variation. This was required to ensure that optimal solutions existed for the problems however it is not necessary here because we will impose conditions (assumptions 1 and 2) so that solutions will exist in these smaller spaces $\mathscr{A}^{n}$ and $\mathscr{L}^{m}$. One of these conditions, assumption 1, could not be used in [6] because it requires $f$ not to behave linearly in $u$ as $u$ gets large.

\section{Construction of the dual problem}

Define the Lagrangian $L$ by

$$
\begin{aligned}
& L(t, x, v)=\inf _{u}\{a(t) x+f(t, u): v=A(t) x+B(t) u+c(t), \\
&C(t) x+E(t) u+d(t) \geqslant 0, u \geqslant 0\} .
\end{aligned}
$$

This corresponds to the primal integrand. From [8] we see that the dual integrand is

$$
\begin{aligned}
M(t, p, s)= & \sup _{x, v}\{s x+p v-L(t, x, v)\} \\
= & \sup _{x, u}\{s x+p(A(t) x+B(t) u+c(t))-a(t) x-f(t, u): \\
\qquad & \quad C(t) x+E(t) u+d(t) \geqslant 0, u \geqslant 0\} \\
= & \sup _{x, u} \inf _{w}\{s x+p(A(t) x+B(t) u+c(t))-a(t) x-f(t, u)
\end{aligned}
$$

In order to gain an explicit form for $M$ we would like to swap around the supremum and infimum operations. Ordinarily if we do this we will obtain something different but we will show in Proposition 1 that in this case the order of the operations essentially does not matter. So in anticipation of Proposition 1 
we will write

$$
\begin{aligned}
& M(t, p, s)=\inf _{w} \sup _{x, u}\{p c(t)+w d(t)+(s+p A(t)+w C(t)-a(t)) x \\
& +(p B(t)+w E(t)) u-f(t, u): w \geqslant 0, u \geqslant 0\}, \\
& =\left\{\begin{array}{rr}
\underset{w}{\inf \sup _{u}\{p c(t)+w d(t)+(p B(t)+w E(t)) u-f(t, u)}, \\
w \geqslant \hat{0}, u \geqslant 0\} \\
+\infty \text { otherwise. } & \text { if } s+p A(t)+w C(t)-a(t)=0,
\end{array}\right.
\end{aligned}
$$

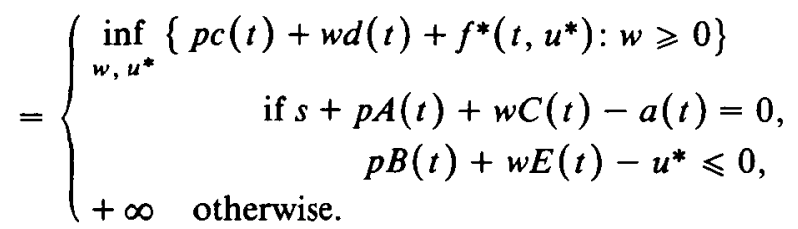

which amounts to the integrand and constraints for (D). What remains, is to verify that we can indeed swap around the supremum and infimum. Let $y=(x, u)$ and with $p$ and $s$ fixed, define

$$
K(y, w)=\left\{\begin{aligned}
s x+p(A(t) x+B(t) u+c(t))-a(t) x-f(t, u) & \\
& +w(C(t) x+E(t) u+d(t)) \quad \text { if } w \geqslant 0, u \geqslant 0, \\
+\infty & \text { if } w \neq 0, \\
-\infty \quad \text { if } u \ngtr 0 . &
\end{aligned}\right.
$$

The order in the definition of $K$ is important. It implies $K(y, w)=+\infty$ if $w \neq 0, u \neq 0$.

\section{Proposition 1. Either}

$$
\sup _{y} \inf _{w} K(y, w)=\inf _{w} \sup _{y} K(y, w)
$$

or the left hand side is $-\infty$ and the right hand side is $+\infty$.

Proof. We always have

$$
\sup _{y} \inf _{w} K(y, w)=\inf _{w} \sup _{y} K(y, w) .
$$

If the left hand side is $+\infty$ then equality trivially holds. Suppose the left hand side is finite then if $\inf _{w} K(y, w)$ is finite we must have

$$
\inf _{w} K(y, w)=K(y, 0)
$$


since $K$ is linear in $w$ on $\mathbf{R}_{+}^{k}$. Hence

$$
\sup _{y} \inf _{w} K(y, w)=\sup K(y, 0) \geqslant \inf _{w} \sup _{y} K(y, w)
$$

which is the reverse inequality to the one above and so equality must hold.

Suppose $\sup _{y} \inf _{w} K(y, w)=-\infty$ then there does not exist a $y=(x, u)$ that satisfies

$$
C(t) x+E(t) u+d(t) \geqslant 0, \quad u \geqslant 0 .
$$

By Gale's Theorem of the Alternative [5, page 33] there exists a $w_{0}$ such that

$$
\begin{gathered}
w_{0} C(t)=0, \quad w_{0} E(t)=0, \\
w_{0} d(t)=-1, \quad w_{0} \geqslant 0 .
\end{gathered}
$$

If $\inf _{w} \sup _{y} K(y, w) \neq+\infty$ there must be a $\bar{w}$ and $\mathrm{a} \bar{u}^{*}$ such that

$$
\begin{gathered}
s+p A(t)+\bar{w} C(t)-a(t)=0, \\
p B(t)+\bar{w} E(t)-\bar{u}^{*} \leqslant 0, \\
\bar{u}^{*} \in \operatorname{dom} f^{*}(t, \cdot), \\
\bar{w} \geqslant 0
\end{gathered}
$$

where $\operatorname{dom} f^{*}(t, \cdot)=\left\{u^{*}: f^{*}\left(t, u^{*}\right)<+\infty\right\}$, the effective domain of $f^{*}(t, \cdot)$. Then for any number $\lambda \geqslant 0, \bar{w}+\lambda w_{0}$ also satisfies those constraints for the same $\bar{u}^{*}$. Hence

$$
\inf _{w} \sup _{y} K(y, w) \leqslant p c(t)+\left(\bar{w}+\lambda w_{0}\right) d(t)+f^{*}\left(t, \bar{u}^{*}\right) .
$$

Letting $\lambda \rightarrow+\infty$ we see that

$$
\inf _{w} \sup _{y} K(y, w)=-\infty
$$

and once again equality holds. Q.E.D.

If the primal problem (P) or the dual problem (D) or both have feasible solutions then we can effectively ignore the anomaly in Proposition 1 and equality will essentially hold, since if (P) is feasible $\sup _{y} \inf _{w} K(y, w)>-\infty$ or if (D) is feasible, then for some $(s, p)$, (in fact all feasible pairs $(s, p)$ ) we will have inf $_{w} \sup _{y} K(y, w)<+\infty$. The case when neither (P) nor (D) is feasible is not of considerable interest.

From the construction of the dual problem, even when we ignore Proposition 1, we will always have,

Theorem 1 (Weak Duality).

$$
\inf \Psi_{P} \geqslant-\inf \Psi_{D}
$$


Proof. See Proposition 2 of [8]. Q.E.D.

The obvious corollaries to this are

COROLlary 1. If there exists a feasible $(x, u)$ such that

$$
\Psi_{P}(x, u)=-\inf \Psi_{D}
$$

then $(x, u)$ is optimal for $(\mathrm{P})$.

COROLlaRY 2. If there exists a feasible $\left(p, w, u^{*}\right)$ such that

$$
\inf \Psi_{P}=-\Psi_{D}\left(p, w, u^{*}\right)
$$

then $\left(p, w, u^{*}\right)$ is optimal for (D).

These are sufficient conditions for optimality, perhaps not very useful, but nevertheless they suffice. Combining the corollaries and using the derivation of the dual problem one can obtain another set of sufficient conditions analogous to the complementary slackness principle of linear programming.

Although weak duality holds for these problems, in general strong duality does not, namely, we cannot say that inf $\Psi_{P}=-$ inf $\Psi_{D}$ unless we add some assumptions to the problems. An example of two problems that exhibit a duality gap are

$$
\begin{gathered}
\min \Psi_{P}(u)=\int_{0}^{1} u^{2}(t) d t \\
\text { subject to } \dot{x}(t)=u(t), \quad x(0)=0, \\
x\left(\frac{1}{2}\right) \geqslant 1, \quad x(t) \geqslant 0, \\
u(t) \geqslant 0, \\
\min \Psi_{D}\left(u^{*}\right)=\int_{0}^{1}\left(u^{*}\right)^{2} / 4 d t \\
\text { subject to } \dot{p}(t)=-w(t), \quad p(1)=0, \\
p(t) \leqslant u^{*}(t), \\
w(t) \geqslant 0 .
\end{gathered}
$$

In this case inf $\Psi_{P}=2$ whereas $-\inf \Psi_{\mathrm{D}}=0$.

\section{Existence and strong duality}

We will now turn to the study of whether the primal and/or dual problems have optimal solutions. The difficulty here lies in the fact that if the controls are bounded in the primal problem by means of the linear constraints then the constraints in the dual problem will not bound the dual controls and vice versa. This follows from Tucker's theorem of the alternative [5, page 29]. In order to show that optimal solutions exist for both problems then, as well as bounding the controls for one of the problems by our choice of $E(t)$ we must also place 
conditions on the other problem to ensure that, although the controls can become as large as they like, if they start to become too large the objective functional will penalise them so that they will not be optimal. Remember, to be a feasible control it must be Lebesgue measurable, and must not contain anything like a delta function for example. We will achieve this by placing a growth condition of the type usually found in existence theory, on the objective functional for the primal problem.

The main result of this section is Theorem 3 which gives details about when the primal and dual problems have equal values and when optimal solutions exist. Following this we give some simple but widely applicable examples of when the conditions required in Theorem 3 are satisfied.

The results in this section follow mainly from [9].

Definitions. The state constraint multifunction $X: T \rightrightarrows \mathbf{R}^{n}$ is given by $X(t)=$ $\{x: \exists u \geqslant 0$ with $f(t, u)<\infty$ and $C(t) x+E(t) u+d(t) \geqslant 0\}$.

The dual state constraint multifunction $\bar{P}: T \rightrightarrows \mathbf{R}^{n}$ is given by $\bar{P}(t)=\left\{p: \exists u^{*}\right.$, and a $w \geqslant 0$ with $f^{*}\left(t, u^{*}\right)<\infty$ and $\left.p B(t)+w E(t)-u^{*} \leqslant 0\right\}$.

Assumption 1. Let $f$ be finite on $T \times \mathbf{R}_{+}^{m}$ (where $\mathbf{R}_{+}^{m}=\{u: u \geqslant 0\}$ ), and satisfy the growth condition

$$
\lim _{\lambda \rightarrow+\infty} \frac{f(t, \lambda u)}{\lambda}=+\infty, \quad \forall t \in T, \forall u \in \mathbf{R}_{+}^{n} \backslash\{0\} .
$$

An example of a function that satisfies this assumption is

$$
f(t, u)=|u|^{2}
$$

whereas the function $f(t, u)=|u|$ does not.

Assumption 2. Let the components of $E(t)$ be $e_{i j}(t)$. Assume the $e_{i j}(t)$ are nonnegative for all $t \in T$ and there exists a number $\delta>0$ such that for each $t$ and $i$ there exists a $j$ such that $e_{i j}(t) \geqslant \delta$.

Proposition 1. Under Assumption (1), $\bar{P}(t)=\mathbf{R}^{n}$ for all $t \in T$.

Proof. The growth condition in assumption 1 implies that $f^{*}\left(t, u^{*}\right)<\infty$ for all $u^{*} \in \mathbf{R}^{n}$ [7, Theorem 13.3]. Hence for any $p$ and any $w \geqslant 0$ we can find a $u^{*} \in \operatorname{dom} f^{*}\left(t, u^{*}\right)$ that majorises $p B(t)+w E(t)$. Q.E.D.

Proposition 2. Under Assumptions (1) and (2), $X(t)=\mathbf{R}^{n}$ for all $t \in T$. 
Proof. Fix an $x \in \mathbf{R}^{n}$. We can choose a $\bar{u} \geqslant 0$ such that

$$
\bar{u}_{i} \geqslant \max _{1 \leqslant j \leqslant k}\left\{-\frac{1}{8}[d(t)+C(t) x]_{j}\right\}, \quad 1 \leqslant i \leqslant m .
$$

Then $E(t) \bar{u} \geqslant-d(t)-C(t) x$ and since $f$ is finite on $\mathbf{R}_{+}^{n}$, this $\bar{u}$ will be feasible. Q.E.D.

To use the results in [9], we must firstly convert (P) and (D) into calculus of variations problems. These will be

$$
\underset{x \in A^{n}}{\operatorname{minimize}} \Phi_{P}(x)=l\left(x\left(t_{0}\right), x\left(t_{1}\right)\right)+\int_{T} L(t, x(t), \dot{x}(t)) d t
$$

and

$\left(\mathrm{D}^{\prime}\right) \underset{p \in A^{n}}{\operatorname{minimize}} \Phi_{D}(p)=m\left(p\left(t_{0}\right), p\left(t_{1}\right)\right)+\int_{T} M(t, p(t), \dot{p}(t)) d t$,

where $L$ and $M$ were defined in $\S 3$.

TheOREM 2. Under Assumptions (1) and (2), both $L$ and $M$ are normal integrands with $L(t, \cdot, \cdot)$ and $M(t, \cdot, \cdot)$ convex for each $t \in T$. Also, for every $x \in \mathscr{A}^{n}$, and $p \in \mathscr{A}^{\prime \prime}$ one has

$$
\begin{aligned}
& \Phi_{P}(x)=\min \left\{\Psi_{P}(x, u): u \in L^{m} \text { feasible for } x\right\}, \\
& \Phi_{D}(p)=\min \left\{\Psi_{D}\left(p, w, u^{*}\right): w \in L^{k}, u^{*} \in L^{m} \text { feasible for } p\right\},
\end{aligned}
$$

where the minima are attained. In this sense $(\mathrm{P})$ is equivalent to $\left(\mathrm{P}^{\prime}\right)$ and $(\mathrm{D})$ is equivalent to $\left(\mathrm{D}^{\prime}\right)$.

Proof. This will follow from the Equivalence Theorem of [10] once we have shown that the inf-boundedness condition of that paper holds for $(P)$ and for (D). For the primal problem, this amounts to showing that for every $t \in T, \alpha \in \mathbf{R}$ and bounded set $S \subset \mathbf{R}^{n} \times \mathbf{R}^{n}$ the set

$$
\begin{array}{r}
\Gamma=\left\{u \in \mathbf{R}^{m}: \exists(x, v) \in S \text { with } f(t, u) \leqslant \alpha \text { where } v=A(t) x+B(t) u+c(t),\right. \\
C(t) x+E(t) u+d(t) \geqslant 0, u \geqslant 0\}
\end{array}
$$

is bounded. But this follows from Assumption 1 and Corollary 14.2.2 of [7].

For the dual problem we must show that for every $t \in T, \alpha \in \mathbf{R}$ and bounded set $S \subset \mathbf{R}^{n} \times \mathbf{R}^{n}$, the set

$$
\begin{gathered}
\Gamma^{\prime}=\left\{\left(u^{*}, w\right) \in \mathbf{R}^{m} \times \mathbf{R}^{k}: \exists(p, s) \in S \text { with } p c(t)+w d(t)+f^{*}\left(t, u^{*}\right) \leqslant \alpha\right. \\
\text { where } \left.s=-p A(t)-w C(t)+a(t), p B(t)+w E(t)-u^{*} \leqslant 0, w \geqslant 0\right\}
\end{gathered}
$$

is bounded. 
Since $f(t, u)$ is finite for all $u \geqslant 0$ then

$$
\lim _{\lambda \rightarrow+\infty} \frac{f^{*}\left(t, u_{0}^{*}+\lambda u^{*}\right)}{\lambda}=+\infty
$$

for any $u_{0}^{*} \in \operatorname{dom} f^{*}(t, \cdot)$ and $u^{*} \in \mathbf{R}_{+}^{n} \backslash\{0\}$. Without loss of generality, we can assume $S$, and therefore $\Gamma^{\prime}$, is convex. If $\Gamma^{\prime}$ is unbounded, then for any $\left(u_{0}^{*}, w_{0}\right) \in \Gamma^{\prime}$ there exists a $\left(\bar{u}^{*}, w^{*}\right)$ such that for all $\lambda \geqslant 0,\left(u_{0}^{*}+\lambda \bar{u}^{*}, w_{0}+\lambda \bar{w}\right)$ $\in \Gamma^{\prime}$. Assumption 2 and the constraints $p B(t)+w E(t) \leqslant u^{*}, w \geqslant 0$ imply that $\bar{u}^{*} \in \mathbf{R}_{+}^{n} \backslash\{0\}$. We also have

$$
\lambda \bar{w} d(t)+f^{*}\left(t, u_{0}^{*}+\lambda \bar{u}^{*}\right) \leqslant \alpha-p c(t)-w_{0} d(t)
$$

which implies

$$
\lim _{\lambda \rightarrow+\infty} \frac{f^{*}\left(t, u_{0}^{*}+\lambda \bar{u}^{*}\right)}{\lambda} \leqslant-\bar{w} d(t)
$$

a contradiction. Therefore $\Gamma^{\prime}$ is bounded. Q.E.D.

Using Theorem 2, in order to determine whether optimal solutions exist for (P) and (D), it suffices to show that optimal solutions exist for $\left(\mathrm{P}^{\prime}\right)$ and $\left(\mathrm{D}^{\prime}\right)$ and for this we can use Theorem 1 of [9]. Two of the conditions that must hold for that theorem to be applicable are:

$\left(\mathrm{C}_{0}\right)$ for each $p \in \mathbf{R}^{n}$ there exist functions $s \in L_{1}^{n}$ and $\alpha \in L_{1}^{1}$ such that $L(t, x, v) \geqslant x s(t)+v p-\alpha(t)$.

$\left(\mathrm{D}_{0}\right)$ for each $x \in \mathbf{R}^{n}$ there exist functions $v \in L_{1}^{n}$ and $\beta \in L_{1}^{1}$ such that $L(t, x, v(t)) \leqslant \beta(t)$.

We will use the following assumptions to ensure that this is the case.

Assumption 3. For each $\gamma \in \mathbf{R}^{m}, \exists u \geqslant \gamma$ and a summable function $\beta$ such that $f(t, u) \leqslant \beta(t)$ a.e.

This generalizes the finiteness assumption of $f$. If $g$ is a summable function then

$$
f(t, u)=g(t)|u|^{2}
$$

satisfies assumption 3 . The conjugate of this function is

$$
f^{*}\left(t, u^{*}\right)=\frac{1}{4} g^{-1}(t)\left|u^{*}\right|^{2}
$$

and if $g^{-1}$ is a summable function then the next asumption will also hold.

Assumption 4. For each $\rho \in \mathbf{R}^{m}, \exists u^{*} \geqslant \rho$ and a summable function $\sigma$ such that $f^{*}\left(t, u^{*}\right) \leqslant \sigma(t)$ a.e.

These assumptions hold, as well, when $f$ is independent of $t$ and satisfies Assumption 1.

Proposition 3. Let Assumptions 1 through 4 hold. Then conditions $\left(\mathrm{C}_{0}\right)$ and $\left(\mathrm{D}_{0}\right)$ also hold. 
Proof. By the corollary to Proposition 4 in [9] it is enough to show that the Hamiltonian

$$
H(t, x, p)=\sup _{v}\{p v-L(t, x, v)\}
$$

is a finite, summable function of $t \in T$ for every $(x, p) \in \mathbf{R}^{n} \times \mathbf{R}^{n}$. From the definition of the Lagrangian,

$$
\begin{aligned}
H(t, x, p)= & \sup _{u}\{p[A(t) x+B(t) u+c(t)]-a(t) x-f(t, u): \\
& C(t) x+E(t) u+d(t) \geqslant 0, u \geqslant 0\} \\
\geqslant & p A(t) x+p c(t)+p B(t) \bar{u}-f(t, \bar{u})-a(t) x
\end{aligned}
$$

where

$$
\bar{u}_{i} \geqslant \operatorname{ess} \sup \max _{t \in T}\left\{-\frac{1}{\delta}[d(t)+C(t) x],\right\}, \quad 1 \leqslant i \leqslant m \text { and } u \geqslant 0 .
$$

The supremum is finite because the components of $d$ and $C$ are essentially bounded. Then assumption 3 implies $H(\cdot, x, p)$ is bounded below by a finite, summable function.

The Hamiltonian can also be expressed as

$$
\begin{aligned}
& H(t, x, p)=\inf _{s}\{M(t, p, s)-s x\} \\
& =\inf _{w, u^{*}}\left\{p c(t)+w d(t)+f^{*}\left(t, u^{*}\right)\right. \\
& \left.\quad+[p A(t)+w C(t)-a(t)] x: B(t)+w E(t)-u^{*} \leqslant 0, w \geqslant 0\right\} \\
& \leqslant p c(t)+f^{*}\left(t, \bar{u}^{*}\right)+p A(t) x-a(t) x
\end{aligned}
$$

where

$$
u^{*} \geqslant \underset{t \in T}{\operatorname{ess} \sup } p B(t)
$$

Assumption 4 implies that $H(\cdot, x, p)$ is bounded above by a finite summable function. Q.E.D.

In order to state our theorem on the existence of optimal solutions, when strong duality also holds, we need some definitions dealing with attainability.

DeFinitions.

$$
\begin{gathered}
C_{l}=\left\{\left(x_{0}, x_{1}\right) \in \mathbf{R}^{n} \times \mathbf{R}^{n}: l\left(x_{0}, x_{1}\right)<+\infty\right\}, \\
D_{L}(t)=\left\{(x, v) \in \mathbf{R}^{n} \times \mathbf{R}^{n}: L(t, x, v)<+\infty\right\} .
\end{gathered}
$$

The set $C_{L}$ of weakly attainable pairs for $(\mathrm{P})$ is the set $C_{L}=\left\{\left(x_{0}, x_{1}\right)\right.$ : $(x(t), \dot{x}(t)) \in D_{L}(t)$ a.e. $\}$. We can similarly define the sets $C_{m}, D_{M}(t)$ and $C_{M}$.

We denote by aff $C$ the affine hull of a set $C$ (the smallest affine set containing $C$ ). If $C$ is convex we denote by ri $C$ the relative interior of $C$ (the interior of $C$ relative to aff $C$ ). 
Our main theorem is

TheOREM 3. (a) If Assumptions 1 through 4 hold and ri $C_{m} \cap$ ri $C_{M} \neq \varnothing$ then $\inf \Psi_{P}=-\inf \Psi_{D}>-\infty$.

In addition, if $(\mathrm{P})$ is feasible then an optimal solution exists for $(\mathrm{P})$.

(b) If Assumptions 1 through 4 hold and ri $C_{l} \cap$ ri $C_{L} \neq \varnothing$ then $+\infty>\inf \Psi_{P}=-\inf \Psi_{D}$.

In addition, if $(\mathrm{D})$ is feasible then an optimal solution exists for (D).

Proof. By Proposition 3 conditions $\left(\mathrm{C}_{0}\right)$ and $\left(\mathrm{D}_{0}\right)$ hold. Combining Theorem 2 with Theorem 1 of [9] yields the result. Q.E.D.

We will now consider cases in which the above conditions dealing with the relative interiors will be valid.

COROllary 3. Let Assumptions 1 through 4 hold. If

$$
\lim _{\lambda \rightarrow+\infty} \frac{l\left(x_{0}+\lambda \bar{x}_{0}, x_{1}+\lambda \bar{x}_{1}\right)}{\lambda}=+\infty, \quad\left(x_{0}, x_{1}\right) \in \operatorname{dom} l,
$$

unless $\bar{x}_{0}=0$ or $\bar{x}_{1}=0$ or both, then ri $C_{m} \cap$ ri $C_{M} \neq \varnothing$ and the results of Theorem 3(a) hold.

Proof. As $\left(\mathrm{C}_{0}\right)$ holds by Proposition 3 , we can apply Corollary 1 of Theorem 3 from [9]. Referring to that paper we see, due to Assumption 1, that

$$
\hat{L}(t, x, v) \geqslant \begin{cases}a(t) x & \text { if } v=A(t) x, C(t) x \geqslant 0, \\ +\infty & \text { otherwise. }\end{cases}
$$

Because of the growth condition on $l$ above, we must have $z\left(t_{0}\right)=0$ or $z\left(t_{1}\right)=0$ or both in the Corollary of [9]. But as $\dot{z}(t)=A(t) z(t)$ as well, the only such arc is the zero arc. Q.E.D.

The growth condition on $l$ in the above corollary usually applies, for example if the primal problem is a fixed endpoint problem

$$
l\left(x\left(t_{0}\right), x\left(t_{1}\right)\right)=\left\{\begin{array}{l}
0 \text { if } x\left(t_{0}\right)=x_{0}, \\
+\infty \text { otherwise, }
\end{array}\right.
$$

or if one of the endpoints, or both, is contained in a bounded set

$$
l\left(x\left(t_{0}\right), x\left(t_{1}\right)\right)=\left\{\begin{array}{l}
h\left(x\left(t_{0}\right)\right) \text { if } x\left(t_{1}\right) \in S_{1}, \\
+\infty \text { otherwise. }
\end{array}\right.
$$

Another simple example where the growth conditions holds is if $l\left(x_{0}, x_{1}\right)=x_{0}^{2}+$ $g\left(x_{1}\right)$. Here $\bar{x}_{0}$ must be zero. For any of these cases, Corollary 3 tells us that if the problem $(\mathrm{P})$ is feasible, then it has an optimal solution and strong duality holds. 
DEFINITION. The recession function $\hat{m}$ for $m$ is

$$
\hat{m}\left(\bar{p}_{0}, \bar{p}_{1}\right)=\lim _{\lambda \rightarrow+\infty} m\left(p_{0}+\lambda \bar{p}, p_{1}+\lambda \bar{p}_{1}\right)
$$

where $\left(p_{0}, p_{1}\right) \in \operatorname{dom} m$.

COROLlaRY 4. Let Assumptions 1 through 4 hold. If

$$
\begin{gathered}
\hat{m}\left(p\left(t_{0}\right), p\left(t_{1}\right)\right)+\int_{T}[p(t) c(t)+w(t) d(t)] d t \leqslant 0, \\
\dot{p}(t)=-p(t) A(t)-w(t) C(t), \\
p(t) B(t)+w(t) E(t) \leqslant 0, \quad w(t) \geqslant 0,
\end{gathered}
$$

has no solutions $p \in \mathscr{A}^{n}, w \in L^{k}$ other than the zero solution, then ri $C_{l} \cap$ ri $C_{L} \neq \varnothing$ and the results of Theorem $3(\mathrm{~b})$ hold.

Proof. As $\left(\mathrm{D}_{0}\right)$ holds, we can apply Theorem 3 of [9]. The definition of $M$ and the growth condition on $f^{*}$ implied by the finiteness of $f(t, \cdot)$ on $\mathbf{R}_{+}^{n}$ allows us to replace

$$
\hat{m}\left(p\left(t_{0}\right), p\left(t_{1}\right)\right)+\int_{T} \hat{M}(t, p(t), \dot{p}(t)) d t
$$

with the above. The result then follows from the equivalence of $\left(b^{\prime}\right)$ and $\left(c^{\prime}\right)$ in that theorem. Q.E.D.

Propositrion 4. Let Assumptions 1 through 4 hold and

$$
l\left(x\left(t_{0}\right), x\left(t_{1}\right)\right)=\left\{\begin{array}{l}
0 \text { if } x\left(t_{1}\right) \in S_{1}, \\
+\infty \text { otherwise, }
\end{array}\right.
$$

where $S_{1}$ is a compact set in $\mathbf{R}^{n}$. Then both the primal and dual problems have optimal solutions and

$$
\min \Psi_{P}=-\min \bar{\Psi}_{D}
$$

Proof. The dual endpoint function is

$$
\begin{aligned}
m\left(p_{0}, p_{1}\right) & =\sup _{x_{0}, x_{1}}\left\{p_{0} x_{0}-p_{1} x_{1}-l\left(x_{0}, x_{1}\right)\right\} \\
& = \begin{cases}\delta_{S_{1}}^{*}\left(p_{1}\right) \text { if } p_{1}=0 \\
+\infty & \text { otherwise }\end{cases}
\end{aligned}
$$

where $\delta_{S_{1}}^{*}\left(p_{1}\right)=\sup \left\{p_{1} x_{1}: x_{1} \in S_{1}\right\}$.

From Corollary 4 , if the zero solution is the only solution to

$$
\begin{gathered}
\dot{p}(t)=-p(t) A(t)-w(t) C(t), \quad p\left(t_{1}\right)=0, \\
p(t) B(t)+w(t) E(t) \leqslant 0, \\
w(t) \geqslant 0
\end{gathered}
$$


then an optimal solution exists (if a feasible one does) to the dual problem (D). For any matrix or vector $H$ let $\|H\|$ denote $\left(\sum_{i, j} H_{i j}^{2}\right)^{1 / 2}$. By Assumption 2, the constraint in Corollary 4 implies

$$
0 \leqslant w_{i}(t) \leqslant \frac{1}{\delta}\|p(t)\| \cdot\|B(t)\|, \quad 1 \leqslant i \leqslant k .
$$

Let $\bar{B}=\operatorname{ess} \sup _{t \in T}\|B(t)\|$. Similarly define $\bar{A}, \bar{C}$. Then

$$
\|\dot{p}(t)\| \leqslant\|p(t)\| \bar{A}+\|p(t)\| \frac{\bar{B} \bar{C}}{\delta} .
$$

Now the only solution to the differential equation

$$
\dot{\rho}(t)=\left(\bar{A}+\frac{\bar{B} \bar{C}}{\delta}\right) \rho(t)
$$

with $\rho\left(t_{0}\right)=0$ is the zero solution. Hence

$$
\|\dot{p}(t)\| \leqslant \dot{\rho}(t)=0 \quad \text { and } \quad p(t)=0 \quad \forall t \in T .
$$

Assumption 2 then implies $w(t)=0 \forall t \in T$. Therefore (D) has an optimal solution, if a feasible one exists. But the same situation applies to (P) (see the comments after Corollary 3 regarding an $l$ of this type). Combining parts (a) and (b) of Theorem 3 we see

$$
+\infty>\inf \Psi_{P}=-\inf \Psi_{D}>-\infty
$$

and since neither problem is infinite, feasible solutions must indeed exist. So both problems have optimal solutions. Q.E.D.

The same result will hold if

$$
l\left(x\left(t_{0}\right), x\left(t_{1}\right)\right)=\left\{\begin{array}{l}
0 \text { if } x\left(t_{0}\right) \in S_{0}, \\
+\infty \text { otherwise }
\end{array}\right.
$$

where $S_{0}$ is a compact set in $\mathbf{R}^{n}$, or for many other types of endpoint functions $l$.

\section{References}

[1] E. P. Bodo and M. A. Hanson, "A class of continuous nonlinear complementarity problems", $J$. Optim. Theory Appl. 24 (1978), 243-262.

[2] W. H. Farr and M. A. Hanson, "Continuous time programming with nonlinear constraints", $J$. Math. Anal. Appl. 45 (1974), 96-115.

[3] R. C. Grinold, "Continuous programming, part two: nonlinear objectives", J. Math. Anal. Appl. 27 (1969), 639-655.

[4] M. A. Hanson and B. Mond, "A class of continuous convex programming problems", J. Math. Anal. Appl. 22 (1968), 427-437.

[5] O. L. Mangasarian, Nonlinear programming (McGraw-Hill, New York, 1969). 
[6] J. M. Murray, "Some existence and regularity results for dual linear control problems", to appear in J. Math. Anal. Appl.

[7] R. T. Rockafellar, Convex Analysts (Princeton University Press, Princeton, N. J., 1970).

[8] R. T. Rockafellar, "Conjugate convex functions in optimal control and the calculus of variations", J. Math. Anal. Appl. 32 (1970), 174-222.

[9] R. T. Rockafellar, "Existence and duality theorems for convex problems of Bolza", Trans. Amer. Math. Soc. 159 (1971), 1-40.

[10] R. T. Rockafellar, "Existence theorems for general control problems of Bolza and Lagrange", Áav. in ivain. 15 (1975), 312-333.

[11] R. T. Rockafellar, "Integral functionals, normal integrands and measurable selections", in Nonlinear Operators and the Calculus of Variations (L. Waelbroeck, ed.), (Springer-Verlag, 1976), 157-207. 